Meta

Journal des traducteurs

Translators' Journal

\title{
L'articulation du texte en anglais et en français
}

\section{Alan Manning}

Volume 37, numéro 3, septembre 1992

URI : https://id.erudit.org/iderudit/003553ar

DOI : https://doi.org/10.7202/003553ar

Aller au sommaire du numéro

Éditeur(s)

Les Presses de l'Université de Montréal

ISSN

0026-0452 (imprimé)

1492-1421 (numérique)

Découvrir la revue

Citer cette note

Manning, A. (1992). L'articulation du texte en anglais et en français. Meta, 37(3), 563-566. https://doi.org/10.7202/003553ar d'utilisation que vous pouvez consulter en ligne.

https://apropos.erudit.org/fr/usagers/politique-dutilisation/ 


\section{L'ARTICULATION DU TEXTE EN ANGLAIS ET EN FRANÇAIS}

\section{DÉFINITIONS}

Amorcer une refflexion sur l'articulation en anglais et en français appelle forcément une définition de l'articulation et de la notion texte.

- L'articulation peut être désignée comme étant le fait de mettre en relation, le plus souvent logique, des mots ou des groupes de mots (Vinay et Darbelnet 1958: 220-232).

- Un texte, nous disent Galisson et Coste (1976: 560), est un ensemble d'énoncés écrits, qui en constitue un discours suivi et un tout spécifique.

On peut donc dire que l'articulation sert à relier tous les éléments d'un texte afin de créer un ensemble cohérent, un peu comme on relie les pièces d'un casse-tête.

\section{ÉTAT DE LA QUESTION}

L'articulation a déjà fait l'objet de plusieurs études, chacune ayant été menée dans une optique différente.

- Ducrot et Anscombre ont abordé l'articulation sous l'aspect «pragmatique» dans la perspective de la logique.

- Charolles a travaillé surtout sur les discours écrits dans l'optique de la «textualité». Leurs analyses portent sur les éléments qui contribuent à rendre un texte cohérent, dont l'articulation.

- Plusieurs études ont été effectuées sur le rôle de la redondance aussi bien dans le discours oral qu'écrit. La notion de redondance est empruntée à la théorie de l'information. Selon cette notion, l'articulation constitue un des éléments qui diminuent le poids de l'information véhiculée dans la communication en ce sens qu'elle n'apporte aucune information nouvelle. D'où sa redondance.

- Hallyday et Hasan analysent la cohésion du texte d'un point de vue linguistique et non de communication. Bien sûr, une large place est faite à l'étude de l'articulation.

- Point n'est besoin de commenter le chapitre de la Stylistique comparée sur l'articulation, tellement il est connu de tous. L'analyse du rôle de l'articulation y est avant tout formelle et linguistique, et confronte le comportement des articulateurs en anglais et en français.

Les trois dernières études sont plus proches de nos préoccupations, car notre objectif consiste à étudier d'un point de vue formel et linguistique l'articulation du texte en anglais et en français. D'ailleurs, c'est parce que la Stylistique comparée est la seule analyse in extenso du rôle de l'articulation qui propose, en plus, un schème cohérent des articulateurs en anglais et en français que nous avons décidé de nous en servir dans notre propre étude.

\section{PROBLÉMATIQUE}

Dans la Stylistique comparée, les articulateurs, que nous appellerons dorénavant charnières, sont rangés, selon leur fonction, en quatre grandes catégories:

Groupe A - type de charnière: explicite, c'est-àdire comprend trois subdivisions :

1. mots-vides, c'est-à-dire les conjonctions de la grammaire traditionnelle, ex. : «aussi», «de plus», «par conséquent», etc.;

2. un membre de phrase (type sémantique), ex.: «comme nous avons déjà vu», «nous passerons maintenant à»;

3. mot-vide $\longrightarrow>$ étoffement dans $L A$ ex. : «Là» $\longrightarrow>$ «In that place».

Groupe B - type de charnière: implicite, c'est-àdire une charnière dont le sens est constitué par rapport à un mot plein ou toute une phrase. ex.: this, these.

Groupe $\mathbf{C}$ - type de charnière : de liaison (ou coordination),

$$
\text { ex. : et, ou, etc. }
$$

Groupe D - type de charnière : zéro, c'est-à-dire les éléments d'un énoncé sont reliés par simple juxtaposition, ex. : II nous fit signe de nous arrêter; nous nous exécutâmes.

Selon le schème proposé par la Stylistique comparée, les chamières se retrouvent en français et en anglais de la manière suivante: 


$\begin{array}{cccc} & \text { Français } & & \text { Anglais } \\ & \text { A } & -> & \text { A } \\ & \text { B } & -> & \text { B } \\ \text { mais } & \text { C } & -> & \text { C } \\ & \text { A } & -> & \text { D } \\ & \text { A } & -> & \text { B }\end{array}$

Aux fins de cet article, nous aimerions ajouter à ces quatre catégories une cinquième catégorie tout à fait particulière, celle qui regroupe les charnières de la ponctuation et de la disposition graphique.

\section{MÉTHODOLOGIE}

Notre étude s'appuie sur deux articles de journal : d'une part, un article intitulé «L'État éclaté», paru dans le journal français Le Monde du 19 novembre 1982; d'autre part, un article intitulé «Damascus is not Beirut», paru dans le journal hebdomadaire britannique The Manchester Guardian Weekly du 12 décembre de la même année. Ce dernier est la traduction de l'article français. Or, deux étudiants diplômés avaient déjà fait sous notre direction une analyse formelle et linguistique de l'articulation dans les deux articles à partir du schème proposé par la Stylistique comparée, sans toutefois s'attarder aux cas où le schème faisait défaut. Notre objectif consiste donc à relever les cas-problèmes, à proposer des explications qui permettent de préciser davantage ce que nous entendons par «traduire».

Notre étude s'articulera ainsi en deux volets: dans un premier temps, nous commenterons des exemples qui témoignent de la justesse du schème proposé par la Stylistique comparée.

Dans un deuxième temps, nous nous pencherons sur des cas où la traduction de certaines charnières semblerait obéir à des critères extra- plutôt qu'interlinguistiques. En conclusion, nous proposerons un cadre d'analyse de l'articulation qui, sans exclure celui présenté dans la Stylistique comparée, doit toutefois s'y superposer.

\section{DISCUSSION}

a) La traduction des charnières françaises en anglais. Les exemples de 1 à 10 correspondent au schème énoncé dans la Stylistique comparée. Its feront l'objet d'un bref commentaire divisé en trois parties :

a) le contexte;

b) type de charnière;

c) solution proposée par la Stylistique comparée.

[1] Les dirigeants de la résistance soutiennent néanmoins, en substance, que le bilan de la guerre du Liban est «globalement positif».

Yet. Palestinian resistance leaders maintain that in fact the outcome of the war in Lebanon was "positive on the whole".

a) Contexte: L'État des fedayin vient de s'effondrer. Les dirigeants palestiniens nient l'ampleur de la défaite.

b) Type de chanière : $A_{1}$ Explicite c) Solution proposée par la Stylistique : traduire par $A_{1}$ en anglais.

[2] À n'en pas douter, «l'État des fedayin» a éclaté. There is no doubt the "fedayin state" has been shattered.

a) Contexte: Vient en début d'un paragraphe. Paragraphe précédent constate que la défaite des Palestiniens est totale.

b) Type de charnière: $A_{2}$ explicite, membre de phrase.

c) Solution proposée par la Stylistique: traduire $\mathrm{A}_{2}$ en anglais.

[3] Témoin cette équipe de télévision américaine venue de New York... An example of this: an American television ovew, which flew in from New York...

a) Contexte: La presse mondiale n'est pas toujours la bienvenue en Syrie. Notre exemple en fournit une illustration percutante.

b) Type de charnière : $\mathrm{B}$, implicite.

c) Solution proposée par la Stylistique: traduire par type $B$ en anglais.

[4] Damas n'est pas et ne sera jamais Beyrouth. Damas is not, and never will be, Beirut.

a) Contexte: La déclaration du président Assad vient appuyer une série de mesures visant à restreindre la liberté d'action des Palestiniens réfugiés en Syrie.

b) Type de charnière: $\mathrm{C}$, simple liaison (coordination).

c) Solution proposée par la Stylistique : traduire par type C.

[5] La plupart n'ont pas de domicile fixe; nombre d'entre eux sont logés chez des proches ou à l'hôtel.

Most of them have no fixed address and many of them are living with relatives.

a) Contexte: Les hauts responsables palestiniens sont en désarroi, et notre exemple en est une illustration.

b) Type de charnière: $\mathrm{D}$, zéro.

c) Solution proposée par la Stylistique : très vague à ce propos. En français, le point-virgule établit un équilibre entre deux propositions. En anglais, le traducteur a «explicité» la fonction par l'emploi d'une charnière de liaison. Donc une simple juxtaposition en français est rendue par une charnière-addition.

[6] Il a alors averti les diverses organisations de la centrale des fedayin qu'il «ne tolérerait aucune provocation»...

He warned the various fedayin organizations that "would not tolerate any provocation"...

a) Contexte: À la suite de l'embuscade que les Palestiniens avaient tendue à une patrouille 
israélienne à la frontière entre Israël et la Syrie, Assad donne un avertissement aux Palestiniens.

b) Type de charnière : $\mathrm{A}$, explicite.

c) Solution proposée par la Stylistique: traduire souvent par type D - charnière zéro.

[7] Un soldat syrien, somnolant sur une chaise, un fusil posé nonchalamment sur les genoux, monte la garde à la porte d'un modeste immeuble, au cour de la ville.

A Syrian soldier, dozing in a chair with his rifle resting negligently in his tap, mounted guard at the entrance to a modest building in the heart of the capital.

a) Contexte : Mise en scène de l'article.

b) Type de charnière : de ponctuation.

c) Solution proposée par la Stylistique: il existe dans la Stylistique maints exemples de cet usage de la virgule et en anglais et en français : la virgule sert à distinguer des propositions et des groupes de mots pour la clarté du contenu. Cette valeur des virgules correspond dans les textes à une liaison. Il est intéressant de remarquer l'anglais with qui remplace en quelque sorte la virgule et vient attester sa valeur de liaison. $\mathrm{La}$ dernière en français semble discutable.

[8] Un jeune homme en civil vérifie l'identité du visiteur avant de lui permettre de pénétrer - qui l'eût cru? - au siège de l'O.L.P.

A young man in civvies checked the visitor's identity before letting him into - who would have believed it? - the PLO headquarters.

a) Contexte: Suit l'exemple [7].

b) Type de charnière : de ponctuation (une ellipse).

c) Solution proposée par la Stylistique: valeur d'ellipse bien attestée dans les deux langues (p. 230). Traduire par le double tiret en anglais.

[9] Paragraphe 3 du texte français et paragraphes $3,4,5$ du texte anglais.

a) Contexte: Annonce une série de mesures prises par Assad à la suite de l'embûche tendue à la patrouille israélienne.

b) Type de charnière : disposition graphique.

c) Solution proposée par la Stylistique: divisez le texte en trois, car l'anglais préfère émettre une seule idée par paragraphe. En ce sens, la division devient elle-même une charnière.

[10] Sous-titres: L'État éclaté : Un bilan globalement positif. Un redoutable bilan.

a) Les sous-titres reprennent en gros l'idée prédominante des paragraphes qui les suivent.

b) Type de charnière : de disposition graphique.

c) Solution proposée par la Stylistique: le français sent davantage le besoin d'annoncer son sujet (propos), l'anglais non. L'anglais aura donc tendance à supprimer les sous-titres.

\section{RÉSUMÉ}

Nous avons jusqu'ici essayé de démontrer que le schème proposé par la Stylistique pour la traduction des charnières en anglais et en français est certainement valable. Il existe, toutefois, comme on l'a vu à l'exemple [5], des cas où la traduction des charnières ne paraît pas relever de tendances linguistiques mais de critères autres.

Voir exemple [1].

Pour la deuxième partie de la discussion, nous ajouterons une $4^{\mathrm{e}}$ catégorie à notre cadre d'analyse des exemples. Nous procéderons donc de la façon suivante: a) contexte; b) type de charnière; c) solution proposée par la Stylistique; d) solution du traducteur.

[11] Voir exemple [1].

d) Solution du traducteur: aurait pu laisser la charnière au milieu de la phrase. Mais il préfère la placer en début de phrase pour bien marquer l'opposition entre cette proposition et celle qui la précède. Notons que la Stylistique n'aborde pas cet aspect de l'articulation. Il est d'ailleurs intéressant de remarquer la modulation d'une autre charnière - en substance : «pour l'essentiel» qui devient in fact: «en réalité», dont le rôle est d'appuyer l'opposition. Enfin, remarquons l'adjonction d'un nouvel élément lexical qui confère à la phrase plus de précision encore: Palestinian.

[12] Voir exemple [2].

d) Solution du traducteur: $y$ va d'une transposition grammaticale cette fois-ci. Il semble avoir voulu donner plus de souplesse à la phrase qui du coup est plus fluide.

[13] Voir exemple [3].

d) Solution du traducteur: reformule la proposition. La charnière est détachée du reste de la proposition par l'adjonction d'une charnière de ponctuation. La nouvelle charnière a pour effet de mettre en relief ce qui la suit. On remarquera également que le segment détaché est reformulé pour faire une proposition indépendante.

[14] Voir exemple [4].

d) Solution du traducteur: en français, les deux propositions - l'une enchâssée - sont mises sur le même plan. Le traducteur a voulu donner plus de «visibilité» à la deuxième proposition en l'encadrant de virgules. En même temps, il parvient à faire ressortir l'opposition entre le présent ( $\mathrm{I}^{\text {re }}$ proposition) et le futur. Ceci est également un exemple intéressant, puisque la charnière zéro est plutôt l'apanage du français.

[15] Ils n'auront plus libre accès aux masse-médias qui leur avaient permis de s'adresser directement à l'opinion arabe et internationale: tandis que les journaux et la radio-télévision du pouvoir 
baasiste se permettent d'occulter, de censurer, voire de tronquer les déclarations et les communiqués des fedayin, les représentants de la presse mondiale ont du mal à exercer leur métier dans un pays où ils ne sont pas toujours les bienvenus. They will not have free access any more to the media which, when they were in Beirut, had enabled them to address Arab and world Opinion directly. While the Syrian media are free to obfuscate, censure or even doctor statements put out by Fedayin organizations, representatives of the world press have a hard time doing their job in a country where they are not always well accepted.

a) Contexte : voir texte.

b) Type de charnière : de ponctuation.

c) Solution proposée par la Stylistique : si la deuxième partie de la phrase est ressentie comme la retombée logique de la première, on traduira pour les deux points en anglais. Cependant, l'usage des deux points en français semble discutable et s'explique uniquement par la valeur de traitement que le journaliste a voulu donner à cette charnière. La deuxième partie de l'énoncé est vue comme une explication de la première. Il $\mathrm{y}$ a introduction de termes qui sont des oppositions explicatives de la première partie de l'énoncé. D'où le choix de charnière.

d) Solution du traducteur: en anglais, il est impossible de maintenir la même opposition, en partie parce que nous ne pouvons reproduire le jeu de mots «permis/permettre». Le traducteur a donc préféré séparer les oppositions en recourant au point.

Ceci démontre l'élément de choix personnel du traducteur. L'on pourrait en déduire que c'est pour des fins de clarté d'exposition que le traducteur a restructuré les phrases.

[16] D'une part, il est crucial pour eux de préserver le peu d'autonomie qui leur reste après la perte du sanctuaire libanais. Ils sont, d'autre part, condamnés à composer avec le pouvoir «fort» de Damas qui est capable de confisquer leur liberté d'action.

For one thing, it is vitally necessary for them to hang on to what little autonomy they are left with after the loss of their Lebanese sanctuary; and for another, they must willy-nilly keep on good terms with the "strong" government of Damascus which could cramp their freedom of action.

a) Contexte : voir texte.

b) Type de charnière: balancement séparé par le point.

c) Solution proposée par la Stylistique: balancement de ce type peut être maintenu en anglais. L'usage du point paraît discutable, mais peut s'expliquer par le fait que l'opposition est ressentie très fortement par le journaliste français comme des idées distinctes mais liées quand même. d) Solution du traducteur: Le traducteur a choisi d'utiliser le point-virgule, charnière qui met les deux énoncés en équilibre. Cet équilibre est accentué par le déplacement de in another au début du deuxième énoncé. Il est à noter que le point-virgule en anglais joue le rôle de charnière d'opposition qui articule deux propositions. C'est un des signes de ponctuation plus forts sur le plan stylistique. Son emploi ici permet de structurer la phrase de façon bien équilibrée à tous points de vue.

Résumé : Nous avons essayé de démontrer qu'un schème d'articulation comme celui proposé par la Stylistique peut servir de guide de façon générale, mais que ce n'est pas la langue qui prend la décision finale, pour ainsi dire.

\section{CONCLUSION}

La décision finale - comment traduire - appartient, évidemment, au traducteur. Il dispose d'une marge de liberté en même temps qu'il doit se plier à un certain nombre de contraintes. Il doit d'abord et avant tout véhiculer le message tout en respectant la (les) fonction (s) du texte et sa population-cible. Autrement dit, il se sert du système linguistique pour rendre le contenu et façonne le contenu selon des éléments extralinguistiques : type de texte, fonction du texte, connaissance de la population-cible ou destinataire. Nous n'avons qu'à regarder les deux derniers exemples pour nous en convaincre. La Stylistique propose des solutions aux problèmes liés à la traduction des charnières. Le traducteur va plus loin. Il y ajoute divers éléments comme la restructuration du message $\left(n^{\circ} 15\right)$ pour clarifier et équilibrer le poids du message. Newmark, dans sa théorie de la traduction communicative, insiste sur le rôle du type et de la fonction du texte, et la nature du destinataire. Nos conclusions tendent à lui donner raison.

ALAN MANNING Université Laval, Québec, Canada

\section{BIBLIOGRAPHIE}

ANSCOMBRE, J.-C. (1983): L'Argumentation dans la langue, Mardaga.

CHAROLLES, M. (1976): «Grammaire de texte Théorie du discours-Narrativité», Pratiques, 11-12, pp. 133-154.

DUCROT, O. (1980): Les Échelles argumentatives, Éditions de Minuit.

GALISSON, R. et D. COSTE (1976) : Dictionnaire de didactique des langues, Hachette.

HALLIDAY, M. A. K. and R. HASAN (1976): Cohesion in English, Longman.

VINAY, J. P. et J. DARBELNET (1958) : Stylistique comparée du français et de l'anglais, Didier. 